\title{
Does lymph node retrieval by grouping according to UICC TNM protocol increase the yield of total number and the number of positive lymph nodes in
pancreaticoduodenectomy specimens?
}

N. K. Samarakoon, L. B. D. J. Siriwikum, S. K. Liyanage, B. A. G. G. Mahendra, R. M. U. S. Rathnayake, D. S. Edirieweera and S. J. de S Hewavisenthi

Department of Pathology, Faculty of Medicine, University of Kelaniya, Sri Lanka

DOI: http://doi.org/10.4038/jdp.v11i2.7719

\section{Introduction}

Lymph node (LN) involvement is an important prognostic factor, reflected in the Union for International Cancer Control(UICC) TNM classification of malignant tumours, where pathological nodal staging is dependent upon the total number of involved LNs in the surgical resection specimen. The objective of this study was to determine the number of LNs retrieved from pancreaticoduodenectomy (PD) specimens and compare them with data from other institutes and determine whether grouping of LNs improves the total yield and the number of positive lymph nodes.

\section{Methodology}

Fourty two pathology reports [grouped (G)-30 and non-grouped (NG)-12] from PD resection specimens between 2011-2016 in a single center were examined. The number of LNs and the method of retrieving LNs, i.e., whether grouped according to the UICC TNM protocol (G) or non-grouped (NG), was established for each case. LN retrieval was done by the post-graduate trainees under the supervision of a consultant. The Wilcoxon signed rank test was used to establish whether there was a statistically significant difference at the $p$ level 0.1 .

\section{Results}

The average $L N$ yield was 14.37. In the $G$ category, mean was 17 (SD - 8.338), and the median was 15 (5-48). In the NG category, mean was 11.75 (SD - 12.45) and the median was 10 (1-31). The mean and median of LNs with metastases in G were 1.56 (SD-2.527) and 5 (0-11) respectively. The mean and median of LNs with metastases in NG were 2.83 (SD-4.460) and $0.5(0-13)$ respectively. The difference in the total number of LNs retrieved in the two groups was statistically significant $(p=0.07)$. However, this was not so with regard to the number of $L N s$ with metastases $(p=0.69)$.

\section{Discussion}

The average number of LNs retrieved (14.37) compared favourably with other studies, 15 in Liverpool UK study (G method), 15.5 in Toronto Canada study and 11-15 in Leiden Netherlands study (whether G/NG were not specified in these studies). The recommendation by the Royal college of Pathologists UK is $15 \mathrm{LNs}$.

\section{Conclusion}

The grouping of lymph nodes improved the total yield significantly. However, it did not affect the number of lymph nodes with metastases. 